\title{
Strates
}

STRATES Matériaux pour la recherche en sciences sociales

Hors-série | 2002

Parcours dans la recherche urbaine, Michel Rochefort, un géographe engagé

\section{Fonctions, responsabilités d'enseignement et de recherche}

*Publications

\section{(2) OpenEdition}

Journals

Édition électronique

URL : http://journals.openedition.org/strates/545

DOI : $10.4000 /$ strates. 545

ISSN : $1777-5442$

Éditeur

Laboratoire Ladyss

Édition imprimée

Date de publication : 1 janvier 2002

ISSN : 0768-8067

Référence électronique

"Fonctions, responsabilités d'enseignement et de recherche », Strates [En ligne], Hors-série | 2002, mis en ligne le 19 mai 2005, consulté le 08 septembre 2020. URL : http://journals.openedition.org/ strates/545; DOI : https://doi.org/10.4000/strates.545

Ce document a été généré automatiquement le 8 septembre 2020

Tous droits réservés 


\title{
Fonctions, responsabilités d'enseignement et de recherche
}

\author{
*Publications
}

\begin{tabular}{|l||l|}
\hline France / Europe \\
\hline \hline 1949-1950 \\
DES de géographie \\
*Michel Rochefort, La plaine \\
d'Autun et ses bordures, études de \\
géographie agraire, mémoire de \\
DES sous la dir. de J. Tricart, \\
Strasbourg, 1949. \\
*Michel Rochefort «L'évolution \\
économique et sociale de \\
Monthelon, Antully et \\
Roussillon " Mémoires Société \\
Eduenne, Autun, 1950. \\
*Michel Rochefort «La \\
pénétration des capitaux dans la \\
campagne autunoise» Revue de \\
géographie de Lyon, 1950. \\
Membre de l'Association des \\
géographes français \\
\hline \hline 1951 \\
Agrégé de l'université, \\
professeur au Lycée Fustel de \\
Coulanges, Strasbourg \\
\hline
\end{tabular}




\section{2}

*Michel Rochefort «À propos des méthodes de la géographie agraire " Vol. Jub. offert à E. de Martonne, Rennes, 1952.

\section{2-1956}

Ministère de la Construction: travaux de préparation du plan d'aménagement régional de l'Alsace

\section{2-1957}

Assistant de géographie à l'université de Strasbourg

\section{3}

*Michel Rochefort (en coll. avec J. Tricart), Initiation aux travaux pratiques de géographie, SEDES, Paris, 1953.

\section{4}

*Michel Rochefort « La structure professionnelle dans les villes d'Alsace " Congrès international de la population, Rome, 1954.

Ministère de la Construction: enquêtes pour l'aménagement de la ville de Molsheim

\section{5}

Membre de l'Union internationale pour l'étude scientifique de la population

\begin{tabular}{|l||l|}
\hline \hline & $\begin{array}{l}1956 \\
\text { *Michel Rochefort «La notion de réseau urbain » Congrès de } \\
\text { l'union géographique internationale, Rio de Janeiro, } 1956 .\end{array}$ \\
\hline \hline 1957 & \\
* Michel Rochefort « Le rôle \\
perturbateur des frontières sur \\
les petites villes en Alsace » Bull. \\
Association de Géographes Français, \\
1957.
\end{tabular}


1957-1958

Attaché de recherche Cnrs

1958

Docteur es Lettres, Michel Rochefort, L'organisation urbaine de l'Alsace,Thèse principale, Faculté de Lettres de Strasbourg, 1958.

1958-1959

Chargé d'enseignement à la faculté de lettres de Strasbourg Centre d'études du Commerce: recherches pour la promotion des centres commerciaux de l'Alsace et l'aménagement de leur zone d'influence

\section{9}

1959

Génie rural: études pour l'aménagement des rivières cévenoles (avec J. Tricart)

*Michel Rochefort, Rapports entre la pluviosité et l'écoulement dans le Brésil tropical, thèse complémentaire, mémoires de l'Institut des Hautes Études d'Amérique Latine, II, 1959.

*Michel Rochefort « Comment la présence d'une grande ville diversifie-t-elle les *Michel Rochefort «L'organisation urbaine de l'Amazonie moyenne » Bull. AGF, 1959.

agglomérations d'une région ?» Bull. AGF, 1959.

*Michel Rochefort « Wasselone, étude dynamique d'une petite ville » Publication Club Vosgien, 1959.

\section{9-1960}

Maître de conférence à la faculté des Lettres de Strasbourg 


\section{0}

*Michel Rochefort, L'organisation urbaine de l'Alsace, Les Belles Lettres, Paris, 1960.

*Michel Rochefort (en coll. avec A. Blanc, E. Julliard, J. Ray), Les régions de l'Est, coll. La France de demain, PUF, Paris, 1960.

\section{0-1961}

Professeur à l'université de Recife, directeur de recherches au Conseil National de géographie de Rio Janeiro, professeur à l'université de Bahia

Membre de l'Association des Géographes brésiliens

Préfecture de Récife: direction de recherches pour l'aménagement du grand Récife

Conseil National de Géographie de Rio de Janeiro :

- les localisations industrielles dans le Sud-Est du Brésil ;

- l'organisation de la vie de relations dans la région de Rio de Janeiro

Commission de Planification de l'État de Bahia: Organisation d'un Institut de recherches des ressources naturelles. Étude pour l'aménagement de la zone de culture du cacao. Le potentiel humain dans le N. E. du Brésil

Détaché auprès du ministère des Affaires étrangères au Brésil, coopération universitaire et technique

Conférence Rio/São Paulo, Bahia/Recife au Congrès national des géographes brésiliens (Mossoro)

*Michel Rochefort, Géographie de la population de l'État de Pernambouc, université de Récife, Brésil, 1962.

\section{1-1964}

Professeur sans chaire à la faculté de Strasbourg

\section{2-1970}

Chargé d'étude au ministère de la Construction (Centre d'Études Économiques et Sociales)

Commissariat général du Plan: direction de recherches pour l'aménagement de l'armature urbaine française. Programme « Métropoles d'équilibre »

* Michel Rochefort « Méthodes d'analyse des fonctions de métropole régionale " colloque franco-polonais, 1963.

*Michel Rochefort, Les fleuves, Que sais-je ? PUF, Paris, 1963.

\section{4-1997}

Professeur à la Sorbonne, chaire de géographie humaine appliquée 


\section{4}

*Michel Rochefort (en coll. avec

J. Labasse) « Le rôle polarisateur des équipements tertiaires supérieurs » congrès de l'Union géographique internationale, Londres, 1964.

*Michel Rochefort (en coll. avec J. Hautreux), Le niveau supérieur de l'armature urbaine française, Commissariat Général du Plan, 1964.

*Michel Rochefort (en coll. avec J. Hautreux) « Les métropoles et la fonction régionale dans l'armature française" Revue Construction-Aménagement, 1964.

1965

*Michel Rochefort

Physionomie générale de l'armature urbaine " Annales de géographie, 1965.

Ministère de l'Éducation nationale : mission

Membre de la délégation française au Congrès mondial de la population à Belgrade

*Michel Rochefort «Relations entre niveau d'équipement et population totale des villes » Congrès mondial de la population

à Belgrade, 1965.

Ministère de l'Éducation nationale : mission

Membre de la délégation française au Colloque francopolonais de Varsovie

*Michel Rochefort « La notion d'armature urbaine " Colloque franco-polonais de Varsovie, 1965.

\section{4}

*Michel Rochefort "Problèmes de la géographie de l'habitat " Revista Brasileira de Geografica, 1964.

Colloque sur les capitales d'Amérique Latine, Toulouse

*Michel Rochefort «L'accroissement démographique des capitales brésiliennes » Colloque sur les capitales d'Amérique Latine, Toulouse, 1964. 


\begin{tabular}{|c|c|}
\hline & $\begin{array}{l}1966 \\
\text { *Michel Rochefort, Géographie de l'Amérique du Sud, Que sais-je } \\
\text { ?, PUF, Paris, } 1966 . \\
\text { *Michel Rochefort « Le rôle régional de Rio de Janeiro » Revue } \\
\text { Civilisation, } 1966 . \\
\text { *Michel Rochefort, "Armature urbaine et réseau urbain", } \\
\text { Revista Geografica, } 1966 .\end{array}$ \\
\hline $\begin{array}{l}\text { 1966-1971 } \\
\text { Réalisation de travaux pour } \\
\text { l'Atelier parisien d'urbanisme et } \\
\text { l'Institut d'aménagement et } \\
\text { d'urbanisme de la région } \\
\text { parisienne: "Localisation et } \\
\text { perspective de déplacement des } \\
\text { activités tertiaires supérieures } \\
\text { dans la région parisienne" }\end{array}$ & $\begin{array}{l}\text { 1966-1971 } \\
\text { Ministère des Affaires étrangères : mission auprès du ministère } \\
\text { du Plan brésilien pour l'organisation de l'étude de l'armature } \\
\text { urbaine; en Uruguay et au Paraguay pour l'organisation de } \\
\text { l'étude de l'armature urbaine; en Argentine, travaux pour le } \\
\text { service d'Aménagement de la province de Buenos-Aires (1971) }\end{array}$ \\
\hline & $\begin{array}{l}\text { 1967-1972 } \\
\text { Ministère de la Coopération, ORSTOM (Office de Recherches } \\
\text { Scientifiques et Techniques d'Outre-Mer). Missions en Côte } \\
\text { d'Ivoire : organisation des études sur les relations villes- } \\
\text { campagnes et les problèmes de réseau urbain } \\
\text { Ministère de l'Éducation Nationale } \\
\text { Membre de la délégation française au congrès de l'Union } \\
\text { géographique internationale à New-Delhi }\end{array}$ \\
\hline $\begin{array}{l}1968 \\
\text { Ministère de l'Éducation } \\
\text { Nationale. Membre de la } \\
\text { délégation française au colloque } \\
\text { franco-hongrois à Budapest } \\
\text { *Michel Rochefort «Difficultés } \\
\text { de l'aménagement d'une } \\
\text { armature urbaine " colloque } \\
\text { franco-hongrois à Budapest, } \\
\text { 1968. }\end{array}$ & \\
\hline $\begin{array}{l}\text { 1969-1985 } \\
\text { Directeur du laboratoire de } \\
\text { Géographie humaine, Paris I, } \\
\text { associé au Cnrs }\end{array}$ & \\
\hline
\end{tabular}




\section{9}

*Michel Rochefort (en coll. avec

R. Guglielmo, E. Dalmasso), Initiation aux sciences économiques à l'usage des géographes, Nathan, Paris, 1969.

*Michel Rochefort (en coll. Avec C. Bidault et M. Petit), Aménager le territoire, Le Seuil, Paris, 1969.

\section{0}

Conférence à Varsovie

\section{9-1974}

Ministère du Plan brésilien: travaux sur l'aménagement de l'État de São Paulo

Ministère des Affaires étrangères: mission auprès du secrétariat d'État à l'Aménagement de l'État de São Paulo Ministère de la Coopération, ORSTOM : missions au Cameroun

\section{0-1971}

*Michel Rochefort «La régionalisation de l'espace au Brésil » Colloque Cnrs de Bordeaux, 1970.

*Michel Rochefort «Typologie fonctionnelle des villes en Afrique tropicale » Colloque Cnrs de Bordeaux, 1971.

\section{1-1972}

Conférence à Bruxelles

Ministère de l'Éducation

Nationale : mission

Membre de la délégation française au Congrès de l'Union géographique internationale à Montréal

\section{3-1985}

Directeur de l'option $3^{\mathrm{e}}$ cycle IEDES (Institut d'Études du Développement Économique et Social): analyse régionale et aménagement de l'espace

\begin{tabular}{|l|l|l|}
\hline $\begin{array}{l}\text { 1973-1980 } \\
\text { Directeur de l'équipe de } \\
\text { recherche IEDES : analyse } \\
\text { régionale et aménagement de } \\
\text { l'espace }\end{array}$ & $\begin{array}{l}\mathbf{1 9 7 3 - 1 9 8 1} \\
\text { Président du Comité technique d'économie-démographie de } \\
\text { l'ORSTOM }\end{array}$ \\
\hline \hline $\begin{array}{l}\text { 1974 } \\
\text { *Michel Rochefort, Économie } \\
\text { géographique et aménagement du } \\
\text { territoire, Cdu-Sedes, Paris, 1974. }\end{array}$ & $\begin{array}{l}\text { Conférences dans différentes villes du Brésil } \\
\text { *Michel Rochefort « La croissance accélérée des villes dans les } \\
\text { pays du Tiers-Monde » Urbanisme, 1975. }\end{array}$ \\
\hline
\end{tabular}

1973

*Michel Rochefort (et al.), L'Amérique du Sud, approche générale,

Ministère de la Coopération, ORSTOM : missions au Cameroun
Bordas, Paris, 1973. 


\begin{tabular}{|c|c|}
\hline $\begin{array}{l}1975 \\
\text { *Michel Rochefort «Le concept } \\
\text { d'armature urbaine" Revue } \\
\text { Urbanisme, } 1975 .\end{array}$ & $\begin{array}{l}\text { 1975-1979 } \\
\text { Ministère des Affaires étrangères: mission auprès du } \\
\text { secrétariat d'État à l'Aménagement de l'État de São Paulo et du } \\
\text { ministère du Plan à Brasilia } \\
\text { Missions auprès de la commission nationale de politique } \\
\text { urbaine du ministère du Plan brésilien }\end{array}$ \\
\hline \multirow[t]{3}{*}{$\begin{array}{l}1976 \\
\text { *Michel Rochefort, Les activités } \\
\text { tertiaires, leur rôle dans } \\
\text { l'organisation de l'espace, Cdu- } \\
\text { Sedes, } 1976 .\end{array}$} & $\begin{array}{l}\text { 1976-1979 } \\
\text { Travaux pour la commission nationale de Politique urbaine du } \\
\text { ministère du Plan brésilien } \\
\text { Conférences à l'université de Tunis }\end{array}$ \\
\hline & $\begin{array}{l}1977 \\
\text { Ministère de l'Éducation nationale } \\
\text { Mission auprès du CERES Tunis } \\
\text { Conférences à l'université d'Oran }\end{array}$ \\
\hline & $\begin{array}{l}1978 \\
\text { Ministère de l'Éducation nationale. Membre de la délégation } \\
\text { française au Colloque franco-indien à New-Delhi : } \\
\text { Michel Rochefort «Systèmes de centres urbains et économie } \\
\text { régionale dans les pays du Tiers-Monde » Colloque franco- } \\
\text { indien, Cnrs, } 1980 \\
\text { Ministère de l'Éducation Nationale : mission auprès du } \\
\text { département de géographie de l'université de Rabat au Maroc } \\
\text { Membre du comité de rédaction de la revue Tiers-Monde }\end{array}$ \\
\hline $\begin{array}{l}\text { 1979-1980 } \\
\text { Présidence du groupe «Villes } \\
\text { dans le territoire " pour la } \\
\text { préparation du VIII Plan, } \\
\text { Commissariat au Plan }\end{array}$ & $\begin{array}{l}\text { Ministère de l'Éducation nationale : mission en Irak, université } \\
\text { de Bagdad }\end{array}$ \\
\hline $\begin{array}{l}\text { 1981-1986 } \\
\text { Président de la Commission } \\
\text { "Recherche urbaine dans les } \\
\text { pays en développement " au } \\
\text { ministère de la Recherche }\end{array}$ & $\begin{array}{l}\text { Mission à l'INAU (Maroc) } \\
\text { Ministère de la Recherche : missions au Brésil }\end{array}$ \\
\hline
\end{tabular}




\begin{tabular}{|c|c|}
\hline $\begin{array}{l}\text { 1982-1986 } \\
\text { Président de la section } \\
\text { "Géographie et Aménagement » } \\
\text { du Conseil Supérieur des } \\
\text { Universités }\end{array}$ & $\begin{array}{l}\text { *Michel Rochefort «Villes moyennes et développement. Le cas } \\
\text { du Brésil " Courrier du CNRS, } \mathrm{n}^{\circ} \text { 57, et Images des Sciences de } \\
\text { l'Homme, 1984. } \\
\text { Ministère des Affaires étrangères : mission au Burkina Faso } \\
\text { auprès de l'Institut panafricain pour le développement } \\
\text { Ministère de la Recherche : missions au Brésil } \\
\text { Ministère des Affaires étrangères: missions de coopération } \\
\text { avec le Brésil (CNPQ, Conseil national de la recherche) } \\
\text { *Michel Rochefort (en coll. avec R. Ladefroux), Rôle de l'État et } \\
\text { discrimination sociale dans l'évolution des services de santé au Brésil, } \\
\text { ministère de la Recherche, 1985. } \\
\text { Ministère de l'Éducation nationale: mission université de } \\
\text { Tananarive }\end{array}$ \\
\hline $\begin{array}{l}\text { 1985-1988 } \\
\text { Vice-Président du Conseil } \\
\text { Scientifique de l'ORSTOM }\end{array}$ & $\begin{array}{l}\text { Ministère des Affaires étrangères: missions dans diverses } \\
\text { universités brésiliennes }\end{array}$ \\
\hline $\begin{array}{l}\text { 1986-1990 } \\
\text { Vice-président de la section } \\
\text { Géographie et Aménagement du } \\
\text { Conseil Supérieur des } \\
\text { Universités }\end{array}$ & $\begin{array}{l}\text { 1986-1988 } \\
\text { Ministère de l'Éducation nationale : missions d'enseignement } \\
\text { au Centre de formation universitaire de Polynésie à Tahiti }\end{array}$ \\
\hline & $\begin{array}{l}1987 \\
\text { *Michel Rochefort «Faut-il créer des villes moyennes dans les } \\
\text { pays du Tiers-Monde ?» Annales du Centre Universitaire, } \mathrm{n}^{\circ} \text { 2, } \\
\text { Tahiti, } 1987\end{array}$ \\
\hline $\begin{array}{l}\text { 1988-1992 } \\
\text { Membre du Conseil scientifique } \\
\text { de l'université Paris I }\end{array}$ & $\begin{array}{l}1988 \\
\text { *Michel Rochefort «Problèmes généraux du logement des } \\
\text { pauvres dans les pays du Tiers-Monde» Revue Tiers-Monde, } \mathrm{n}^{\circ} \\
\text { 116, } 1988 .\end{array}$ \\
\hline $\begin{array}{l}1989 \\
\text { *Michel Rochefort, Deux siècles } \\
\text { d'aménagement du territoire. Deux } \\
\text { siècles d'histoire de France, Paris, } \\
1989 .\end{array}$ & $\begin{array}{l}1989 \\
\text { *Michel Rochefort «Villes, réseaux urbains et régions dans les } \\
\text { pays du Tiers-Monde. De la fiction à la réalité » in Mélanges } \\
\text { offerts à B. Kayser, Toulouse, } 1989 .\end{array}$ \\
\hline
\end{tabular}


1990-1993

Président du comité scientifique du département Recherches pour le développement du ministère de la Recherche et de l'Espace

Membre du groupe de synthèse Prospective et Territoires à la Datar.

\section{2-1995}

Membre de la section $39 \mathrm{du}$ Comité national du Cnrs

*Michel Rochefort « Métropoles et fragmentation des espaces " in Habitat et villes, l'avenir en jeu, L'Harmattan, Paris, 1992.

1993

*Michel Rochefort « Le Parcours scientifique du programme » in Mutations économiques et urbanisation, La Documentation française, Paris, 1993.

*Michel Rochefort (avec F. Ascher, Brams et al.), Les territoires du futur, Paris, Éditions de l'Aube, 1993.

*Michel Rochefort «Des métropoles d'équilibre aux métropoles d'aujourd'hui » in Métropoles en déséquilibre, Economica, Paris, 1993.

\begin{tabular}{|l|l|l|}
\hline \hline & $\begin{array}{l}\text { 1994-2002 } \\
\text { Ministère des Affaires étrangères : coopération universitaire. } \\
\text { Département d'urbanisme de l'École Nationale d'Architecture } \\
\text { et d'Urbanisme de Tunis : missions dans le cadre des accords } \\
\text { bilatéraux }\end{array}$ \\
\hline \hline $\begin{array}{l}\text { * Michel Rochefort, Dynamique de } \\
\text { l'espace français et aménagement } \\
\text { du territoire, L'Harmattan, Paris, } \\
1995 .\end{array}$ & \begin{tabular}{|l} 
Conférences à l'université de Tunis \\
\hline
\end{tabular} \\
\hline
\end{tabular}




\begin{tabular}{|c|c|}
\hline $\begin{array}{l}\text { Depuis } 1997 \\
\text { Président du Conseil } \\
\text { d'administration de l'Institut } \\
\text { Français d'Urbanisme } \\
\text { Professeur émérite à l'université } \\
\text { de Paris I }\end{array}$ & $\begin{array}{l}\text { 1997-2002 } \\
\text { Travaux pour les services d'urbanisme brésiliens : Fortaleza, } \\
\text { Recife, Campo Grande, Campinas } \\
\text { Ministère des Affaires étrangères: missions dans diverses } \\
\text { universités brésiliennes } \\
\text { Collaboration avec le département d'Unicamp (Campinas) et de } \\
\text { Fortaleza (UFCE) }\end{array}$ \\
\hline & $\begin{array}{l}1998 \\
\text { *Michel Rochefort, Redes e Sistemas. Ensinando sobre o Urbano e a } \\
\text { Regiâo, Hucitec, Sào Paulo, } 1998 .\end{array}$ \\
\hline & $\begin{array}{l}2000 \\
\text { *Michel Rochefort, Le défi urbain dans les pays du Sud, } \\
\text { l'Harmattan, Paris, } 2000\end{array}$ \\
\hline
\end{tabular}

